\title{
Structural Modifications of Proteins During Aging
}

\author{
Ari Gafni, PhD
}

$\mathrm{A}$ ging is a fundamental phenomenon that affects somatic cells whose manifestations, however, may differ from one cell type to another. While most of us possess an intuitive understanding of what aging is, and can tell a young person from an old one, a precise definition of aging and its relationship to development has been surprisingly difficult to develop. The phenomenon of senescence can be defined descriptively as the highly complex ensemble of deteriorative processes that slowly, and irreversibly, increase the susceptibility of an organism to disease and predispose it to death. By emphasizing those processes that lead to a decline in the ability of the organism to cope with environmental stresses, this definition allows one to intuitively, though somewhat arbitrarily, separate aging from development (the latter process installing, and enhancing, an organism's ability to adapt to its environment and to respond to changes in it). The drawback of this very general definition of aging, however, is that it gives no clue as to the fundamental biological processes that regulate, or are involved in, the aging process and provides no indication of the mechanisms by which these processes manifest themselves.

A number of hypotheses to explain the origin of biological aging and to provide more mechanistic insight have been developed and are able to be tested by experiment. These have been described in a number of recent reviews and research publications. ${ }^{1-6}$ The aim of the present review is to provide a somewhat more focused description of one aspect of aging, the age-related changes in proteins, thus summarizing the current knowledge of the aging phenomenon at its most elementary level, the molecular level .

Proteins are macromolecules that serve to execute, regulate, and mediate nearly all biological functions in the cell as well as in the intercellular matrix. The complexity of life processes requires that hundreds, even thousands, of different proteins be present in a living cell at any given moment, each performing a specific task (a typical example being the catalysis of a single chemical reaction within a sequence of reactions that together carry out a biological process). To ensure that only reactions beneficial to the organism occur to any significant extent, proteins need not only be very efficient and specific in their function but must also possess the ability to be regulated by external stimuli (for example by becoming phosphorylated in response to signals from hormones, etc.)

From the Institute of Gerontology and Department of Biological Chemistry, The University of Michigan, Ann Arbor, Michigan.

Supported by Grant AG09761 from the National Institute on Aging.

Address correspondence to Ari Gafni, PhD, Institute of Gerontology and Department of Biological Chemistry, University of Michigan, 300 North Ingalls, Ann Arbor, MI 48109-2007.

so that their activities can be turned on or off upon demand. To achieve these diverse properties, proteins have evolved to be extremely modular macromolecules. They are comprised of linear chains of covalently linked combinations of the 20 natural amino acids (a typical polypeptide chain being a few hundred residues long). Each protein has a specific, unique, sequence that determines its identity and biological nature. This sequence is assembled on the ribosome by translating the message contained in the sequence of nucleotides of the messenger RNA encoding the protein which, in turn, has been transcribed from the message encoded by the sequence of the DNA that comprises the gene specific for that protein.? The one-to-one correspondence between the sequence of nucleotides in a gene and the sequence of amino acids in the protein encoded by that gene is commonly referred to as the genetic code.

The successful synthesis of a polypeptide chain is, however, not sufficient to generate biological activity. To become a functional protein the nascent polypeptide chain that comes off the ribosome has to fold into a very precise, compact, three-dimensional structure. Failure to do so yields a defective, usually nonfunctional, protein. It is generally believed, based on a variety of experimental results and theoretical arguments, that all the information needed to produce the native structure of a protein is contained in the sequence of amino acids in its polypeptide chain. ${ }^{8,9}$ The self organizing process, in which this one-dimensional message is translated into a three- dimensional structure, is termed protein folding $^{10-12}$ and is frequently referred to as the second half of the genetic code. A basic strategy of folding is believed to be the production of partially folded intermediates that contain local structural motifs (secondary structure) and which, being flexible and relatively unstable, continue to "fine-tune" their structure to achieve the more stable native state. This process is schematically depicted in Figure 1. It is pertinent to note, however, that although protein folding has been studied for more than 30 years, it is still not fully understood, and this process continues to pose one of the major unresolved problems of contemporary biology. Moreover, we now know that most proteins in the cell are assisted in their folding by a special class of helper proteins, the molecular chaperones, whose role in directing the folding to produce the biologically functional structure (possibly by inhibiting competing pathways that lead to misfolded, inactive, species) is currently being pursued vigorously. ${ }^{13-15}$

Because the sequence of amino acids determines the ultimate structure, and, hence, the functional competence of a protein, it is not surprising that even small changes in this sequence sometime have profound effects on the biological activity. When the substituted amino acid is one that is 


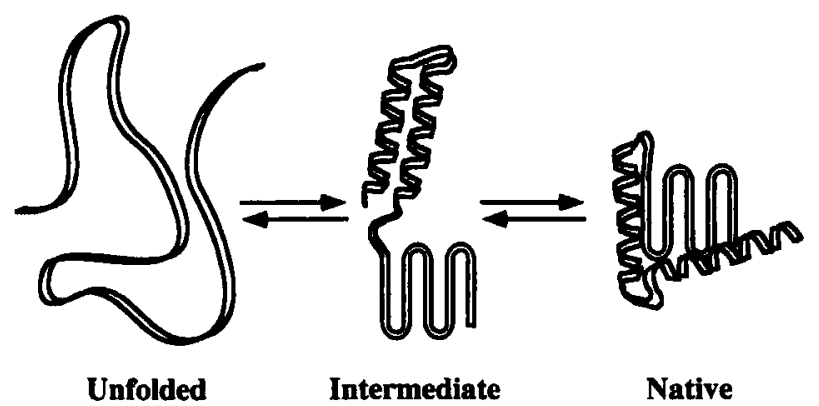

Figure 1. A schematic depiction of the unassisted folding of a protein in vitro. The process begins when the unfolded polypeptide chain, which is devoid of ordered three-dimensional structure and of biological function, rapidly collapses to generate a compact, but flexible, "molten globule" intermediate where elements of secondary structure (alpha helices and beta-sheet domains) have already formed but have not yet interacted to create the tertiary, native structure of the protein. The latter precisely folded and more rigid structure subsequently evolves through a "fine-tuning" process. In the cell protein folding is assisted by a special class of proteins, the molecular chaperones. However, both in vitro and in vivo the folded structure is believed to be determined uniquely by the sequence of amino acids in the polypeptide chain, and since it is usually only marginally stable, even slight changes in this sequence will usually produce a distinctly different structure.

essential for the biological function of the protein, such effects are obvious; however, what is less commonly appreciated is that in many cases, mutations replace residues that are not involved directly in the biological function but that are rather crucial for competent folding or for the stabilization of the folded state. Indeed, the failure of mutant proteins to fold correctly is one of the major causes for their abnormal behavior associated with genetic diseases. ${ }^{16-19}$ It is pertinent to note, however, that structural changes may also develop in protein molecules that have initially folded correctly, leading to a loss or a modification in their biological activities. A number of processes can yield such behavior, including the introduction of covalent modifications (oxidation, glycation, deamidation, methylation, etc.), the isomerization of specific amino acid residues within the protein (cis-trans isomerization of proline residues, racemization of aspartyl residues), or a change in the structure of the folded state of the protein. Examples of such alterations can be found in proteins isolated from old organisms and will be presented in the discussion that follows.

The presence of modifications in proteins isolated from tissues of old animals relative to those isolated from young controls has been well documented, and this topic has been covered in several reviews. ${ }^{20-25}$ These modifications have been found to involve the functional properties, the stability, as well as the structural integrity of these proteins. Old proteins (i.e., those found in tissues of old animals) often, though not always, display a reduction in their biological activity, ${ }^{26-28}$ a modified heat inactivation pattern reflecting alterations in their stability, ${ }^{29-31}$ and frequently possess a modified affinity to antibodies raised against the young form of the protein, a reflection of changes in structural integrity. ${ }^{32,33}$ The fact that, as a rule, those proteins whose activity is affected by aging, display reduced (rather than enhanced) biological competence is not surprising as evolution has strongly selected for each protein a structure that possesses a high biological activity, and a change in the former is likely to reduce the latter. Because most intracellular proteins turn over rather quickly (within hours to days), there is less evolutionary pressure for high stability, and changes in this parameter with aging are less unidirectional. Indeed, it is well documented that proteins isolated from old tissues can display either increased or decreased stabilities; examples of these two behaviors are given in Figure 2 .

The changes observed in the biological activity associated with a given protein in the cell may, in principle, be caused by a decline in the efficiency of protein synthesis (i.e., a smaller quantity of protein being made), by alterations in the fidelity of the synthesis of the protein (i.e., errors in the amino acid sequence), or by posttranslational modifications in the protein (attributable either to chemical alterations or to changes in the folding). A decline in protein synthesis with aging, while potentially of great general importance, does not involve changes in structure and is, therefore, outside the scope of the current review. The appearance with aging of alterations in the fidelity of protein synthesis have, in the past, received much attention, as such alterations were the basis for Orgel's Error Catastrophe theory. ${ }^{34,35}$ This theory was based on the assumption that the low level of errors introduced into protein sequences during normal synthesis will tend to increase exponentially with advancing age because some of the
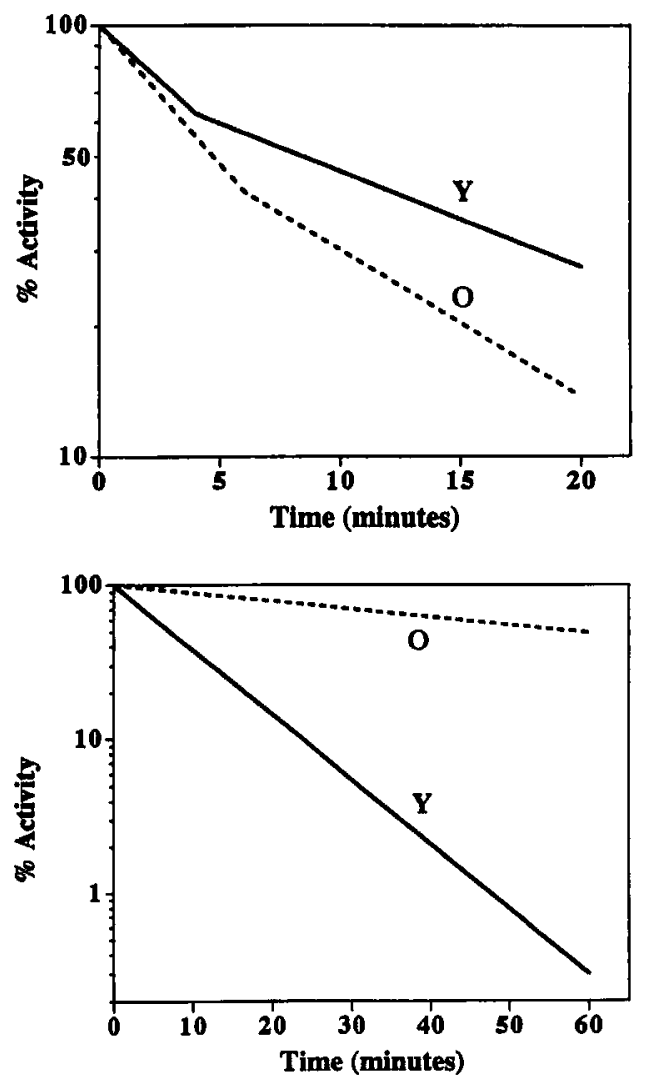

Figure 2. The effects of aging on the heat stability of two proteins isolated from rat muscles. The upper box presents the kinetics of inactivation of glyceraldehyde-3-phosphate dehydrogenase samples isolated from young $(\mathrm{Y})$ and old $(\mathrm{O})$ rats upon incubation at $46^{\circ} \mathrm{C}$. The old enzyme is clearly more heat labile. The lower box presents similar data obtained for phosphoglycerate kinase incubated at $44^{\circ} \mathrm{C}$ revealing, in contrast, a dramatic increase in the heat stability of the old form of this enzyme. 
erroneous proteins initially randomly created will be enzymes involved in protein synthesis. Defects in these proteins will result in an increased level of ill-produced new proteins, and this vicious cycle, where the errors are amplified in each consecutive generation of protein, would lead to catastrophic conditions for the cell. Orgel's theory stimulated a burst of experimental work searching for modified proteins in aging cells and, while many were discovered, no evidence for error amplification was found. More specifically, all the evidence gathered thus far points to postsynthetic modifications as the origin of protein aging, in stark contrast to the main tenet of the Error Catastrophe hypothesis. These postsynthetic alterations are the topic for the discussion below.

\section{CLASSIFICATION OF AGING-RELATED PROTEIN MODIFICATIONS}

Broadly defined, the observed aging-related modifications in proteins fall into two categories: covalent and conformational. Both types of modifications lead to structural changes and, as a rule, to alterations in the biological activity of a protein; however, there is an important distinction between them. Conformational information is erased when the protein is unfolded, and modifications of this type may, therefore, be removed by subjecting the old protein to an unfolding-refolding protocol as will be described below. Covalent modifications, in contrast, are more permanent because they change the identity of the affected amino acids, and this modified sequence, as a rule, will refold into the altered structure. Both covalent and conformational alterations tend to predispose a protein to proteolysis, and it is the decline in the latter process with aging that may be a major reason for the observed increase in the level of modified proteins. It is also of interest to mention that since these age-related modifications are post synthetic, they may all be expected to be more prevalent in long lived proteins relative to those with short dwell times in the tissue.

In the discussion that follows, several specific mechanisms that introduce aging-related covalent modifications in proteins will be described. These have been found to alter both the structural and functional properties of the protein involved. The most prevalent covalent modifications are introduced by oxidation, glycation, or by deamidation and its related reactions. This section will be followed by a discussion of the origin of conformational differences between the young and old forms of a protein, which can reflect either a failure to fold correctly because of modified conditions in the old cell or the development of conformational transitions subsequent to an initially correct folding.

\section{Oxidation}

Oxidative free radicals are produced constantly in the cell as byproducts of normal metabolic processes. These highly reactive species can rapidly interact with, and damage, various cellular components (proteins, nucleic acids, lipids). Indeed, it has been estimated that between 10,000 and 100,000 DNA bases are modified by oxygen free radicals in an average cell every day. ${ }^{36}$ Although cells have defense mechanisms against free radical attack, including those provided by chaperone-proteins as $\alpha$-crystallin, ${ }^{37}$ this protection is not perfect, and a low level of oxidation occurs continuously. Moreover, there is evidence that the efficiency of protection against free radical attack may decline with advancing age, leading to an increased rate of damage accumulation.
Indeed, inasmuch as some of the enzymes involved in oxidative damage control may themselves be affected by free radical oxidation, one would expect the rate of such damaging reactions in the cell to increase with age in an everaccelerating process conceptually akin to the one postulated by the Error Catastrophe theory. Evidence for this type of behavior has been found, and will be discussed below.

The potential role of oxidative free radicals in both aging and disease has been recognized for many years. As early as 1956, Harman proposed his now famous "free radical theory of aging." 38,39 Although a large number of studies have addressed this issue since then, ${ }^{40-4.3}$ our understanding of the role of oxidative stress in aging is still incomplete, and the question of whether oxidation of cellular components is a causative factor in aging or a result of this process brought about by a decline in cellular defense mechanisms has not been fully answered. It is clear, however, that some proteins in old organisms are modified substantially by oxidation. Support for this statement is provided both by the observation that proteins from old animals contain covalent modifications that are clearly the result of oxidation reactions and by the ability to mimic the age-related modifications in proteins by their exposure to oxidative conditions in vitro. ${ }^{44-48}$ From the functional point of view, the two characteristics of a protein that are most noticeably affected by oxidation are the biological activity and the heat stability. The former, when affected, invariably decreases whereas the latter can change in complex ways and sometimes increases. The appearance of modifications involving oxidation in a protein marks this protein for degradation by a number of cellular proteinases. ${ }^{49,50}$ The accumulation of oxidized proteins during aging is believed to be the result not only of the accelerated rate of oxidation but, as importantly, of the age-related decline in the efficacy of these proteinases. ${ }^{50,51}$

One of the prominent age-related oxidative modifications in proteins is the increase in carbonyl content, a product of the oxidation of several amino acid side chains, including proline, arginine, and lysine, as well as of oxidation reactions that lead to the cleavage of peptide bonds. ${ }^{52} \mathrm{~A}$ schematic depiction of carbonyl formation by this reaction is given in Figure 3. Indeed, several researchers have demonstrated that

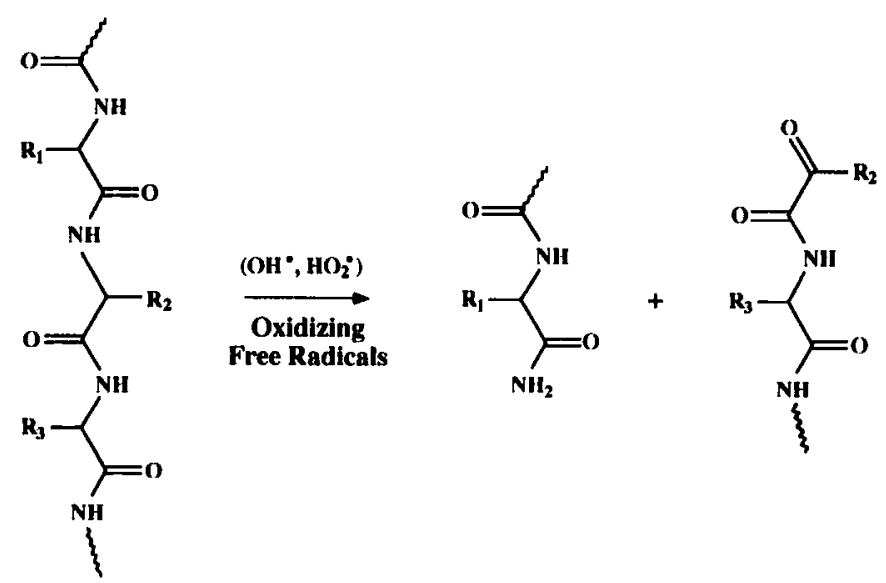

Figure 3. One of the common results of the oxidation of a protein by reactive free radicals is the cleavage of the polypeptide chain, which is accompanied by the introduction of new carbonyl groups. 
the carbonyl content of proteins in a large number of different tissues, as well as in whole body homogenates, increases exponentially with age. ${ }^{53,54}$ It has been estimated that about 30 to $50 \%$ of total cellular proteins in an old animal may be oxidized, ${ }^{44}$ a range typical of the reduction in the catalytic activity observed for many enzymes in old organisms. It is important to note here that carbonyl groups are also introduced into proteins (though transiently) during glycation (discussed in the next section) where a long-lived intermediate, the Amadori product, contains this group (see Fig. 4). Protein glycation thus adds to the pool of carbonylcontaining proteins in a way that is affected by disease (diabetes) and, probably, by aging.

Although the carbonyl content of proteins can be determined experimentally ${ }^{55}$ and has, therefore, been documented and studied in some detail, it is pertinent to mention that the oxidation of some amino acid residues does not lead to the production of these functional groups. Carbonyls are, therefore, not the only result of free radical-mediated protein damage that occurs in aging. For example, histidine oxidation produces aspartate or asparagine, proline oxidation produces glutamate, and cysteine oxidation yields disulfide bonds; in none of these cases are carbonyls generated. ${ }^{56}$ Oxidation of the aromatic amino acid residues phenylalanine, tyrosine, and tryptophan can produce their respective hydroxy derivatives, again generating no carbonyls. It is, clear, therefore, that the carbonyl content of a protein does not reflect the full extent of potential oxidative damage.

As mentioned previously, the oxidation of proteins often leads to discernible changes in their biological and physical properties, including changes in stability, in the ability to be recognized by proteolytic enzymes, and in some spectroscopic signatures. Although oxidation may alter only a very limited number of amino acid residues in a protein directly, sometimes only one, the observed effects may reflect structural alterations that encompass more global protein structure. Thus, it should be appreciated that often it is the oxidation of residues that are not a part of the active site of an enzyme and that do not, therefore, participate in catalysis, that brings about the observed oxidation-related effects. In this respect the alteration of an amino acid residue by oxidation resembles the effect of a point mutation in that it modifies the code for the folded state of the protein. The difference is that the modification in this code caused by a mutation in the gene is incorporated into the nascent polypeptide and, hence, affects the proteins' folding process, whereas oxidation (say of a proline to glutamate) occurs subsequent to the original folding, and its structural consequence is caused by the proteins' readjustment to the introduction of a new group into its prefolded structure. Whether this new structure is identical to the one that would have been generated by folding of the premodified polypeptide chain is an interesting question that has not been carefully studied. It would also be of interest to characterize the structural modifications introduced into proteins in response to amino acid oxidation by comparing the $\mathrm{X}$-ray-derived structures of the original and the oxidized protein forms. This, also, has not been attempted yet.

\section{Protein Glycation}

Glycation, or nonenzymatic glycosylation, of proteins is a complex reaction, depicted schematically in Figure 4. The reaction begins when a carbonyl group of a sugar (frequently glucose) reacts with an amino group on a protein (usually a

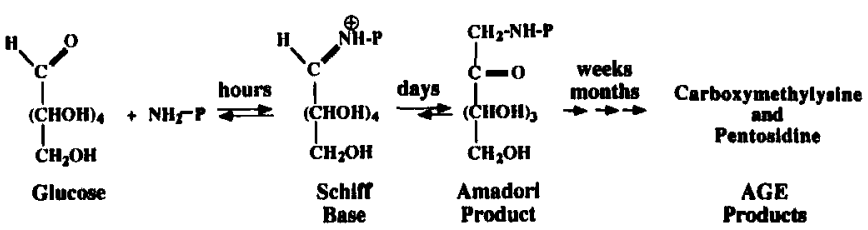

Figure 4. The protein glycation reaction. The carbonyl group of a sugar (here glucose) reacts with a free amino group on the protein to form a Schiff base. This relatively rapid and reversible reaction is followed by the slower, but still reversible, Amadori rearrangement. The free carbonyl group of the Amadori product subsequently undergoes a series of interactions that lead to the slow and irreversible production of the advanced glycosylation end products, including cross-linked protein species.

lysine or the N-terminal amino group) to form a Schiff base. This derivative reacts further, by an Amadori rearrangement, to produce a ketoamine, which is subsequently crosslinked and converted by a complex pathway to advanced glycosylation end products (AGE-products), which are fluorescent, brown pigments. ${ }^{57,58}$ Although not displayed explicitly in Figure 4, oxidation by molecular oxygen plays an important role in glycation in two major ways: (1) it is involved in some of the steps leading to the formation of the AGE-products, ${ }^{59,60}$ and (2) oxidation of the participating sugar can produce reactive dicarbonyls (such as glyoxal) that interact rapidly with amino groups on the protein to ultimately produce N-carboxymethyl amino-acid derivatives (most frequently from lysine). The term autoxidative glycosylation has been coined to describe these reactions. ${ }^{61}$

The time scales involved in these protein modifications have been studied both in vitro and in vivo, and whereas the formation of the Schiff base can occur within a few hours under conditions like those found in the cell, the Amadori product typically takes several days to form, and the AGEproducts develop relatively slowly over a period of weeks to months. ${ }^{62}$ Unlike the Schiff base and the Amadori product, however, both of which are formed by reversible reactions and are thus present at steady state levels, the AGE-products contain irreversible modifications and tend to accumulate over time. If the target protein is one that has a long dwell time in the cell, the extent of glycation may become very significant. Because the degree of glycation increases over time, it is not surprising that its products are enhanced significantly with aging (and even more so in diabetic subjects whose glucose levels are elevated, thereby speeding up the process).

Extracellular matrix proteins tend to be longer-lived than their intracellular counterparts, and are, therefore, generally more markedly altered by glycation. Indeed, in almost all species studied so far, an aging-related increase in the level of glycated extracellular proteins has been observed. One of the best studied cases is collagen, where a build up with age of glycated lysines has been documented in a number of studies. $^{63-66}$ The crosslinking of collagen type I by glycation was shown to induce an expansion of the molecular packing ${ }^{67}$ and to covalently cross-link this protein to soluble plasma proteins, ${ }^{68-70}$ possibly contributing to the narrowing of the vascular lumen. In basement membrane collagen, as well as in laminin, the AGE-products were found to interfere with normal associations and with self assembly of these proteins. ${ }^{71,72}$ Glycation-induced abnormalities in the extracellular matrix were found to alter both the structure and the 
function of intact vessels and have been implicated as a contributing factor in atherosclerosis. ${ }^{73}$ An age-related increase in the level of glycation of the very long lived eye-lens crystallin has been reported to occur in bovine lenses ${ }^{74}$ but, surprisingly, not in human or rat lenses. ${ }^{75,76}$ Thus, the reaction may be species-dependent. It is important to mention that some intracellular proteins, whose turnover rate is slow, are also susceptible to AGE-product formation. Thus, red blood cell hemoglobin, whose dwell-time in human erythrocytes is ca 120 days, is glycosylated measurably, and the extent of modification increases significantly in diabetes. ${ }^{77}$ It is interesting to note that the small heat-shock protein, alpha crystallin, has recently been shown to effectively protect a number of proteins against glycation in vitro. ${ }^{78-80}$ Whether this protein affords a similar protection to cellular proteins in vivo remains to be tested.

Although the relatively high turnover rate of most intracellular proteins makes them less likely to form AGEproducts, these proteins can react with sugars to produce significant amounts of the more rapidly forming Amadori product. Such a modification was found to occur in liver alcohol dehydrogenase in vivo. ${ }^{81}$ Several recent reports reveal that the glycolytic enzyme glyceraldehyde-3-phosphate dehydrogen (GAPDH) isolated from rabbit muscle or from erythrocytes is also partially glycated. ${ }^{82,83}$ While the chemical identity of the glycation product was not determined, it was found to be quite stable as glycated GAPDH could be isolated and studied. This may indicate that glycation went beyond the Amadori product. The activity of glycated GAPDH was found to be reduced relative to that of the native enzyme, and modifications to its tertiary (but not the secondary) structure were found that were assigned to the active site domain. ${ }^{82}$

\section{Deamidation, Racemization, and Isomerization}

The three postsynthetic, protein-modifying reactions in this class all involve asparagine residues. In deamidation, an asparagine is hydrolyzed to produce aspartate and ammonia. Since aspartate is ionized at physiologic $\mathrm{pH}$, this reaction introduces one negative charge into a protein for each deamidation event. In a series of studies, Gracy and coworkers have demonstrated that deamidation increases with aging in a number of proteins and that in both triose-3-phosphate isomerase and glucose-6-phosphate isomerase the electrostatic repulsion among the negative charges produced destabilizes the enzymes and make them more susceptible to proteolysis. $^{84-87}$

Two by-products of the deamidation of asparagine residues in proteins are isoaspartate and $\mathrm{D}$-aspartate. In the production of isoaspartate, shown in Figure 5 , the side-chain carboxyl group becomes connected in the peptide bond, freeing the alpha carboxyl. The generation of $\mathrm{D}$-aspartate is caused by a racemization (the conversion of the native $\mathrm{L}$ amino acid into its optical antipode). A detailed mechanism for asparagine deamidation, isomerization, and racemization was developed by Clarke and coworkers, ${ }^{88,89}$ who demonstrated that in a protein the rates of these reactions depend both on the amino acid that follows the asparagine residue in the sequence and on the three-dimensional structure of the protein, the latter imposing conformational restrictions on these reactions. It is pertinent to mention that, as a rule, racemization of amino acids is a slow process under physiologic conditions and that aspartic acid, which is the most

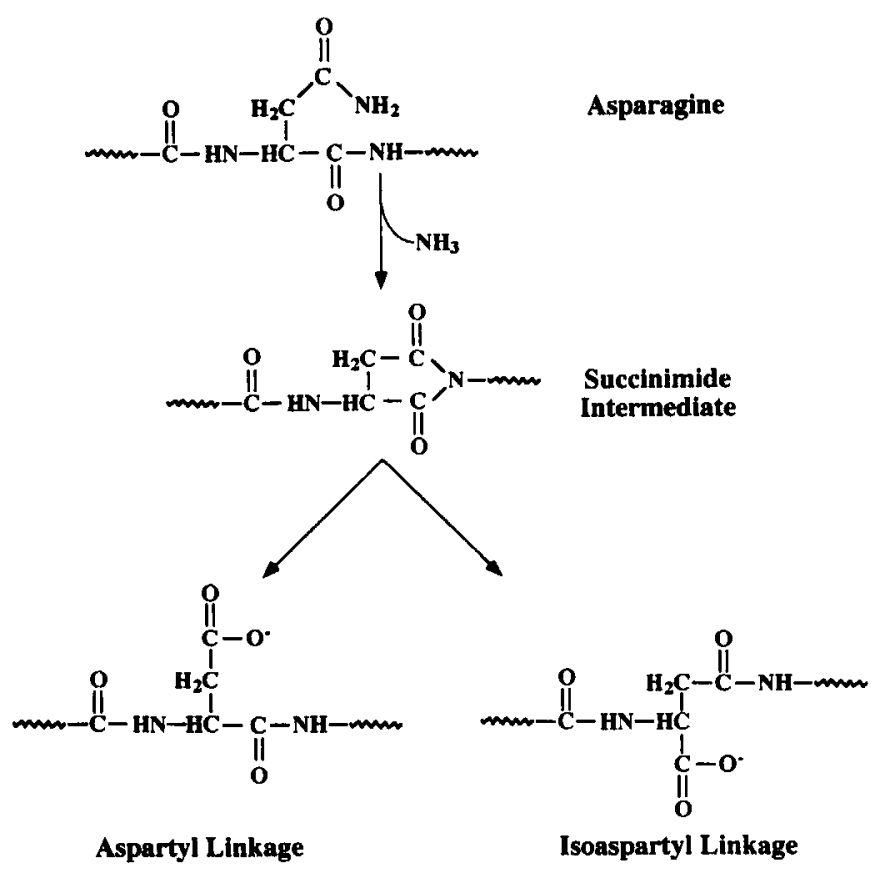

Figure 5. The deamidation of an asparaginyl residue in a protein proceeds in two steps. First, a succinimide intermediate is produced from asparagine in a reaction that is driven by the release of ammonia. The succinimide then undergoes hydrolysis by water, a reaction that can yield either an aspartyl or an isoaspartyl product. In both cases, a new negative charge is generated on the protein; however, the production of isoaspartyl also introduces a non-native linkage into the polypeptide chain.

susceptible to this reaction of all the natural amino acids, only undergoes the transition at a rate of about $0.14 \%$ per year. It is, therefore, to be expected that even in old tissues only a relatively small fraction of Asp residues are modified. Indeed, the accumulation of modified aspartate is only significant in long-lived proteins and has been documented in the eye lens, where protein turnover is almost nonexistent, and in red blood cells where the dwell-time of proteins is several months..$^{9-92}$ Thus, while isomerization and racemization of asparagine can lead to protein modification, their contribution to the pool of altered proteins is relatively small and will not, as a rule, alter most cellular proteins, whose turnover rate is too high to be affected.

\section{CONFORMATIONAL ALTERATIONS AND AGGREGATION}

\section{Age-Related Conformational Alterations}

Because the biological activity of a protein depends critically on the precise three-dimensional structure of its folded state, it is not difficult to envision that any alteration in this structure may have profound effects on the proteins' biological activity. The hypothesis that many of the age-related declines in functional properties of proteins are attributable to conformational modifications was first put forward by Rothstein, ${ }^{93-95}$ who suggested that this behavior may be caused by the longer dwell-time of proteins in the cells of old animals. Strong, though not conclusive, evidence in support of conformational changes as the origin of the age-related modification in a number of proteins was subsequently provided. Thus, Sharma and Rothstein ${ }^{96}$ demonstrated that the 
young and old forms of the glycolytic enzyme enolase (isolated from young and old nematodes, respectively), which display different activities, share the same amino acids sequence; however, when these two enzyme species were unfolded by guanidine: $\mathrm{HCl}$ (a strong denaturant of proteins) and then allowed to refold, they formed an identical structure similar to that of old enolase. Strong evidence was also found for conformational modifications as the origin of the observed aging effects in rat kidney maltase $e^{33}$ and in rat muscle GAPDH. ${ }^{27}$ None of these studies, however, provided direct, conclusive, evidence that there were no other changes in the old forms of these proteins and, hence, that purely conformational alterations were indeed responsible for the effects seen.

Direct evidence for the role of conformational changes in the age-related modifications in a protein was provided for the glycolytic enzyme phosphoglycerate kinase (PGK). This enzyme has been found to become modified in rat skeletal and cardiac muscle, as well as in brain and liver, during aging. ${ }^{97-99}$ A search for covalent modifications detected none $^{100}$; hence, experiments to test for noncovalent structural changes were conducted. In these experiments, schematically depicted in Figure 6, samples of both young and old PGK were unfolded extensively by guanidine: $\mathrm{HCl}$ to erase any differences in the folding pattern of the two proteins. When the denaturant was removed, allowing the proteins to refold and resume their biological activity, it was found that both became identical and behaved as young PGK. ${ }^{101,102}$ This rejuvenation of old PGK by unfolding-refolding provides conclusive evidence that the aging-related modifications
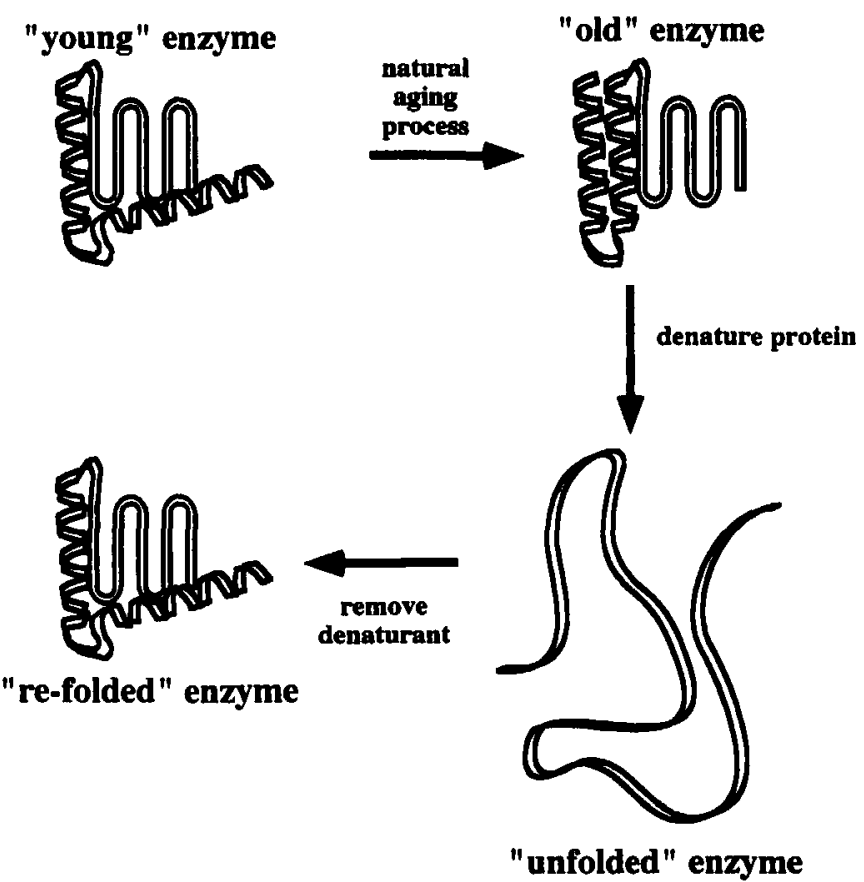

Figure 6. A schematic depiction of age-related structural modifications in a protein where the alterations are solely conformational. The modified tertiary structure usually results in a deficient biological activity though the stability of the protein may actually increase. To test whether the age-related modifications are purely conformational, the old form of the protein can be extensively unfolded, using a strong denaturant, followed by refolding, which should regenerate the native young form of the protein. affect only the conformation of this protein. Although the precise nature of the structural modifications in old PGK is still to be determined, experiments that simulated the aging process of this protein in vitro, producing an enzyme species identical to that found in old tissues, indicated that a temporary oxidation of two cysteine residues to form a disulfide bridge serves as an intermediate step in the process. This model gained strong support from the observation that young PGK in which one of these cysteines has been covalently blocked by methylation becomes resistant to in vitro aging. ${ }^{103}$

Support for the hypothesis that structural modifications are more prevalent in old PGK because of its longer dwell time in the tissue relative to that in young animals, where its turnover rate is high, was provided by experiments in which endurance-trained rats were used. ${ }^{104}$ Exercise-induced damage to muscle proteins triggers de novo protein synthesis and, hence, was expected to shorten the dwell time of proteins in these cells. Indeed, when PGK samples from young, sedentary old, and endurance-trained old rats were compared, the enzymes from the young and the trained old rats were found to be similar and very distinct from the old enzyme from sedentary old animals. Moreover, the old protein could be converted to its young counterpart by an unfolding-tefolding protocol as described above. Thus it appears quite likely that the age-related modifications observed in PGK are purely conformational, develop after the protein has folded to its native state, and are prevalent in old tissues because the rate of turnover of the protein is reduced (under sedentary conditions, but can be enhanced by exercise). It should, however, be emphasized that no direct proof of the longer dwell-time of PGK in old rat tissues has been provided and that other changes in the old cell (a reduction in the amount of protection against conformational changes, for example) could possibly (though less likely) also explain the data.

\section{Amyloid Formation}

The native folded state of soluble, globular proteins, as a rule, places the hydrophobic amino acid residues in the interior and brings only polar residues into contact with the aqueous solvent. Exposure of "sticky" domains rich in hydrophobic amino acid residues leads to protein aggregation and precipitation, a common fate of misfolded proteins. The fact that age-modified PGK stays soluble and monomeric indicates that the structural alterations do not place such hydrophobic domains on the surface of this protein. In contrast, a large number of age-associated diseases and pathologies are characterized by the deposition in tissues of aggregates composed of conformationally modified proteins. What is typical of these deposits, termed amyloid, is that they form fibrillar structures of variable length and with a diameter of 60 to $100 \mathrm{~A}$, in which the individual protein molecules assume a predominantly beta-sheet type secondary structure and assemble into a characteristic cross-beta repeat structure. ${ }^{105} \mathrm{~A}$ model of the mechanism of formation of these structures is depicted schematically in Figure 7, though it is important to mention that this process is still not understood completely. In a recent review, Kelly lists 16 proteins with a high propensity to form amyloid and notes that they display little sequence or structural homology. ${ }^{105}$ The similarity among amyloidogenic proteins is, thus, not in their native folded structure (they are, in fact, not amyloidogenic in their normal state) but rather in their tendency to undergo a 


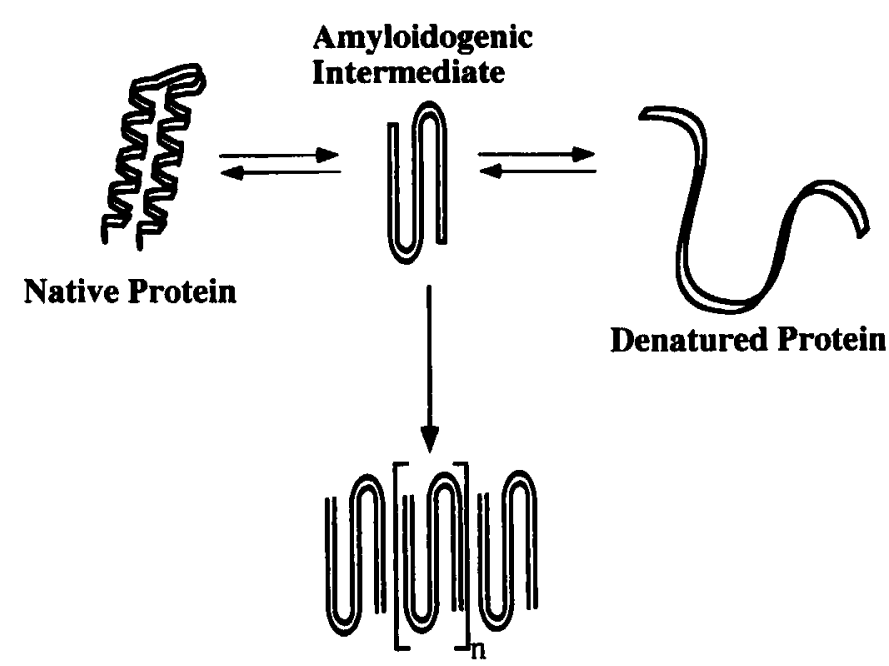

Amyloid

Figure 7. A schematic presentation of the conversion of a correctly folded native protein into an amyloid deposit. The transformation involves the formation of a "sticky" intermediate characterized by a high content of beta-sheet secondary structure, which has a propensity to organize into high molecular weight aggregates that form long, fibrillar structures. The high degree of molecular alignment in these fibrils causes them to display strong birefringence when stained by certain dyes (as Congo Red), which is one of the hallmarks of amyloid deposits.

conformational modification and to acquire amyloidogenicity. A comparison of the evolution of amyloid deposits in different diseases indicates that the process does not follow a uniform pathway but rather is specific to the protein and the disease involved. Thus, in some cases, the amyloidogenic species may be an erroneously produced fragment of a protein or a cleavage peptide that is part of the normal degradation process of the protein, whereas in other cases, the amyloid is formed from the intact protein in a "spontaneous" process, and in yet other cases, the aggregation of the otherwise normal protein is triggered by infection with the diseased form of the protein. Examples for proteins in the first category include fibrinogen fragments, whose deposition is responsible for hereditary renal amyloidosis, ${ }^{106}$ serum amyloid A, whose fragment is involved in secondary systemic amyloidosis, ${ }^{107}$ and the amyloid precursor protein whose cleavage product, beta-amyloid, forms amyloid deposits in the brain of Alzheimer's disease patients. ${ }^{108}$ To the second category belong proteins such as the pancreatic hormone amylin, whose amyloid deposits are prevalent in non-insulindependent diabetes ${ }^{109,110}$ and transthyretin, which features in senile systemic amyloidosis. ${ }^{105}$ The class of infectious amyloidogenic proteins includes the family of prion proteins that are deposited in the brain in a number of diseases including scrapie and Creutzfeldt-Jacob disease. ${ }^{11-113}$

Many of the amyloidogenic diseases show a pronounced age-dependence in that their prevalence increases rapidly towards the end of the life span, reflecting the age-dependent propensity of the proteins involved to undergo the conformational modifications. Possibly the best known of these diseases is Alzheimer's disease, which is characterized by the deposition of amyloid fibrils in the brain that are surrounded by dead or dying neurons. ${ }^{108}$ As mentioned earlier, these fibrils are composed mostly of the beta-amyloid peptide, a
40-43 amino acids fragment of the much larger amyloid precursor protein (APP). The conformational changes that convert the soluble peptide to its amyloidogenic form involve a transformation from a predominantly alpha-helical structure, which is nonaggregatable, to a mostly beta-sheet structure, which is highly aggregatable. These conformational transitions have been studied extensively and although they are, as yet, not fully understood, the contributions of specific electrostatic, as well as hydrophobic, interactions have been delineated, and important structural details have been derived by NMR, CD, and infrared spectroscopies. ${ }^{114-117}$ The beta-amyloid peptide has been shown to exist in normal brain tissue, but what causes the initiation of its deposition in some, but not all, persons is unknown. There is, however, compel ling evidence that these deposits are highly neurotoxic ${ }^{118}$

The deposition of amyloid composed of the 37 amino acids peptide amylin in the pancreas of patients with noninsulin-dependent diabetes mellitus (NIDDM) also involves the conversion of the peptide's secondary structure from a predominantly alpha helical one to a beta-sheet structure. ${ }^{119,120}$ While this system has not been studied in as much detail as the beta-amyloid one, two amino acid residues in amylin, in positions 28,29 in this peptide's sequence, were clearly implicated as key factors in the aggregation. This assignment is based on the comparison of the sequences of human amylin, which is highly amyloidogenic, with those of cat amylin, which is moderately amyloidogenic, and with rat amylin, which does not aggregate. The two serine residues occupying these positions in human amylin were found to determine the amyloidogenic propensity of this species. ${ }^{121}$ However, our understanding of the changes in the cellular environment responsible for amylin aggregation, as well as of the relevance of the amylin deposits in diabetes, is still sketchy.

In some individuals, the thyroxine transporter plasma protein transthyretin (TTR) undergoes conformational modifications that result in its aggregation and deposition as amyloid in the heart, leading to congestive heart failure. This condition, termed senile systemic amyloidosis, usually develops around the age of 80 years and is thus clearly an agingrelated disease. In its native state, TTR is a homotetrameric protein $^{122}$; however, under conditions that cause its dissociation into monomeric subunits, the protein undergoes a conformational change and becomes highly amyloidogenic $^{17,19,123,124}$. Mutants of TTR isolated from individuals with the familial form of the disease (which is much more aggressive and displays an early onset) were found to form less stable protein tetramers and revealed a strong correlation between tetramer instability and amyloidogenicity as discerned both from fibril formation in vitro and from the age of onset of the disease in vivo. ${ }^{123}$ Based on these results, as well as on extensive biophysical studies, Kelly and coworkers have proposed a structure for the amyloidogenic intermediate of TTR in which the tertiary structure is modified but the secondary structure remains native. ${ }^{105}$ As in the amyloidogenic diseases described above, the structural and mechanistic studies of fibril formation, while very instructive, do not provide a clear answer to the question of why the process is so strongly age related. Some possible reasons will be discussed below.

\section{Possible Reasons for the Age-Related Increase in Misfolded and Aggregated Proteins}

All age-related conformational modifications in proteins described in the previous section involve the conversion of a 
correctly folded protein into a conformational isomer. In some cases, this altered protein stays monomeric and soluble, albeit with modified properties (as the old forms of PGK and GAPDH); however, in many cases the new structure has a high propensity to self assemble into amyloid deposits. Several factors may account for the enhanced accumulation of the two types of conformationally modified proteins in old cells:

1. The process of protein folding, as seen in any reaction, is driven toward the minimum in the free energy where the system reaches equilibrium and ceases to change. However, the biologically active (native) form of a protein may be associated with a structure which, though transiently stable, does not represent the equilibrium state, and, given enough time, the protein continues to fold to more stable conformations. A striking example of this type of behavior is provided by plasminogen activator inhibitor-1 (PAI-1), a protein involved in the dismantling and removal of blood clots. The biologically active form of this protein is unstable and converts rapidly and spontaneously to a stable but inactive (latent) conformation. Although PAI-1 may be an extreme case, many cellular proteins (including PGK and GAPDH) share this trait, and their longer dwell time in tissues of old animals allows this conversion to occur to a significantly higher level than in young tissues. In other cases, e.g., in prion-associated diseases, a similar conformational conversion may be triggered (catalyzed) by infection with the diseased form of the protein.

2. As discussed in previous sections, there exists a large body of work demonstrating a progressive increase, with aging, in the level of proteins that have been covalently modified by reactions such as glycation and oxidation. The polypeptide chain, thus permanently altered, will, in principle, encode a different folded structure for the protein, which in the majority of cases will be functionally deficient. This altered conformation may also expose "sticky" domains and thus promote aggregation. Such an increase with aging in the concentration of the amyloidogenic form of a protein may be responsible for the amyloid formation by the Alzheimer's peptide $^{125,126}$ as well as by proteins involved in other amyloidogenic diseases.

3. While the information needed for the correct folding of a protein is believed to be contained in its amino acid sequence, it has become evident in recent years that the folding of most proteins in the cell does not occur independently but is assisted and catalyzed by auxiliary proteins. Some of these helper-proteins, such as protein disulfide isomerase and peptidyl prolyl isomerase, are enzymes that catalyze the formation of the correct disulfide bonds within proteins and the cis-trans isomerization of prolyl peptide bonds (two intrinsically slow reactions which, when not accelerated, tend to clog the folding pathway). Another important group of folding-assistants are the molecular chaperones. These are proteins that help direct the folding along the correct pathway by inhibiting off-pathway processes and interactions. At the high concentration of proteins in the cell, these chaperone proteins play a crucial role as they reversibly bind to temporarily exposed "sticky" segments of the polypeptide chain (generated during folding or unfolding) and thus inhibit their aggregation, ${ }^{127,128}$ a process that would otherwise lead to the appearance of protein precipitates, called inclusion bodies, in the cell. ${ }^{129}$ A pronounced induction of chaperone protein production is a major organismal response to stressful conditions that damage cellular proteins, thereby triggering a burst of de novo protein synthesis and, hence, increasing the demand for chaperones. An age-related decline in the ability of cells to induce chaperone protein production in response to stress has been documented in a number of studies ${ }^{130-133}$ and whereas the origins of this deficiency are still being sought, it appears likely that the structural fidelity of cellular proteins may be affected and that this deficiency can lead to the observed increase in the prevalence of amyloidogenic diseases with aging.

\section{CONCLUSION}

More than 30 years have passed since Orgel proposed his Error Catastrophe theory in an attempt to provide a molecularly based explanation for aging. While later disproven, this hypothesis prompted the search for age-modified proteins that led, several years later, to the discovery of the first example of such species. ${ }^{26}$ We now know that many, though not all, proteins are modified in the tissues of old animals. More importantly, all the evidence thus far gathered indicates that these modifications are introduced by post-synthetic events and are not the result of deficiencies in the protein synthesizing machinery. A number of mechanisms that lead to protein modifications have been identified and studied, some introducing covalent alterations into the polypeptide chain with others affecting the fidelity of the folding pattern of the protein. The reduced ability of cells in old organisms to respond to stressful conditions by elevating the levels of chaperone proteins may feature in this age-related accumulation of misfolded proteins. Whereas the question of whether modified proteins are at the forefront of the aging process or its victims has not been answered, it is clear that their accumulation in old tissues can be highly detrimental. Indeed, an increasing number of devastating diseases has been linked to the presence of structurally modified and functionally defective proteins. Some of these diseases are strongly associated with aging and are characterized by the appearance of amyloid deposits composed of misfolded, aggregated proteins in the diseased tissue. A search for the molecular basis of these diseases, and for successful intervention strategies, is currently underway in a number of laboratories. This is a particularly timely effort because with the projected increase in life expectancy, the number of people affected by diabetes, Alzheimer's disease, etc., is expected to increase rapidly, and the importance of strategies to treat - or better yet, inhibit these diseases cannot be overstated.

\section{REFERENCES}

1. Vijg J, Wei JY. Understanding the biology of aging: The key to prevention and therapy. J Am Geriatr Soc 1995;43:426-434.

2. Martin GR, Austad SN, Johnson TE. Genetic analysis of aging: Role of oxidative damage and environmental stresses. Nature Genet 1996;13:25-34.

3. Martin GR, Danner DB, Holbrook NJ. Aging-causes and defenses. Annu Rev Med 1993;44:419-429.

4. Rose MR. Evolutionary Biology of Aging. New York: Oxford University Press, 1991.

5. Masoro EJ, Austed SN. The evolution of the anti-aging action of dietary restriction. J Gerontol 1996;51A:B387-391.

6. Johnson TE, Lithgow GJ, Murakami S. Hypothesis: Interventions that increase the response to stress offer the potential for effective life prolongation and increased health. J Gerontol 1996;51A:B392-395.

7. Stryer L. Biochemistry. New York: WH Freeman, 1995.

8. Anfinsen CB. Principles that govern the folding of protein chains. Science $1973 ; 181: 223-230$.

9. Richards FM. The protein folding problem. Sci Am 1991;264:54-57.

10. Creighton TE, ed. Protein Folding. New York: WH Freeman, 1992. 
11. Pain RH, ed. Mechanisms of Protein Folding. New York: Oxford University Press, 1994.

12. Ptitsyn OB. Protein folding overview: A determinable but unresolved problem. FASEB J 1996;10:3-5.

13. Ellis RJ, van der Vies SM. Molecular chaperones. Annu Rev Biochem 1991;60:321-347.

14. Buchner J. Supervising the fold: Functional principles of molecular chaperones. FASEB J 1996;10:10-19.

15. Fllis RJ, Hartl FU. Protein folding in the cell: Competing models of chaperonin function. FASFB J 1996;10:20-26.

16. Thomas PJ, Qu BH, Pedersen PL. Defective protein folding as a basis of human discase. Trends Biochem Sci 1995;20:456-459.

17. McCutchen SI., Colon W, Kelly JW. Transthyretin mutation Leu-5.5-Pro significantly alters tetramer stability and increases amyloidogenicity. Biochemistry 1993;32:12119-12127.

18. Bruce D, Perry DJ, Borg JY et al. Thromboembolic disease due to thermolabile conformational changes of antithrombin Rouen-Vl. J Clin Invest 1994;94:2265-2274.

19. MiCutchen SL, Lai Z, Miroy Gi et al. Comparison of lethal and non-lethal transthyretin variants and their relationship to amyloid disease. Biochemistry 1995;34:13527-1.3536.

20. Gafni A. Altered protein metabolism in aging. Annu Rev Gerontol Geriatr 1991;10:117-131.

21. Gafni A. Age-related effects in enzyme metabolism and catalysis. Rev Biol Res Aging 1990;4:315-336.

22. Rattan SIS, Derventzi A, C.lark BFC. Protein synthesis, posttranslational modifications, and aging. Ann NY Acad Sci 1992;663:48-62.

23. Stadtman ER. Protein modification in aging. J Gerontol 1988;43:B112120.

24. Stadtman ER, Oliver CN, Starke-Reed PE, Rhee SC. Age-related oxidation reaction in proteins. Toxicol Ind Health 1993;9:187-196.

25. Rothstein M. Age-related changes in enzyme levels and enzyme properties. Rev Biol Res Aging 1985;2:421-433.

26. Gershon $\mathrm{H}$, Gershon D. Detection of inactive enzyme molecules in aging organisms. Nature 1970;227:1214-1218.

27. Gafni A. Age-related modifications in a muscle enzyme. In: Modifications of Proteins During Aging. RC. Adelman, EE Dekker, eds. New York: Alan R Liss, 1985, pp 19-39.

28. Cordillo E, Ayala A, F-Lobato $M$ et al. Possible involvement of histidine residues in the loss of enzymatic activity of rat liver malic enzyme during aging. J Biol Chem 1988;263:80.53-8057.

29. Reiss U, Rothstein M. Heat labile isozymes of isocitrate lyase from aging Turbatrix aceti. Biochem Biophys Res Commun 1974;61:1012-1016.

30. Sharma HK, Prasanna HR, Rothstein M. Altered phosphoglycerate kinase in aging rats. J Biol Chem 1980;255:5043-5050.

31. Gafni A. Molecular origin of the aging effects in glyceraldehyde-3phosphate dehydrogenase. Biochim Biophys Acta 1983;742:91-99.

32. Dovrat A, Scharf J, Eisenbach I, Gershon D. Glucose-6-phosphate dehydrogenase molecules devoid of catalytic activity are present in the nucleus of the rat lens. Exp Eye Res 1986;42:489-496.

33. Reiss U, Sacktor B. Monoclonal antibodies to renal brush border membrane maltase: Age-related antigenic alterations, Proc Natl Acad Sci USA 1983;80:3255-3260.

34. Orgel LE. The maintenance of the accuracy of protein synthesis and its relevance to aging. Proc Natl Acad Sci USA 1963;49:517-521.

35. Orgel LE. The maintenance of the accuracy of protein synthesis and its relevance to aging: A correction. Proc Natl Acad Sci USA 1970;67:1476.

36. Ames BN, Sigenaga MK, Hagen TM. Oxidants, antioxidants and the degenerative diseases of aging. Proc Natl Acad Sci USA 1993;90:7915-7922.

37. Wang $K$, Spector A. Alpha-crystallin can act as a chaperone under conditions of oxidative stress. Invest Ophthalmol Vis Sci 1995;36:311-321.

38. Harman D. Aging: A theory based on free radical and radiation chemistry. J Gerontol 19.56;11:298-300.

39. Harman D. Free radicals in aging. Mol Cell Biochem 1988;84:155-161

40. Carncy JM, Carncy AM. Role of protein oxidation in aging and in ageassociated neurodegenerative distases. Life Sci 1994;55:2097-2103.

41. Agarwal S, Sohal RS. Relationship between aging and susceptibility to protcin oxidative damage. Biochem Biophys Res Commun 1993;194:12031206.

42. Dean RT, Gebicki J, Gieseg $S$ et al. Hypothesis: A damaging role in aging for reactive protcin oxidation products? Mutat Res 1992;275:387-393.

43. Smith CD, Carney JM, Tatsumo T et al. Protein oxidation in aging brain. Ann NY Acad Sci 1992;663:110-119.

44. Stadtman ER. Rolc of oxidized amino acids in protein breakdown and stability. Mcthods Enzymol 1995;258:379-393.

45. Davies KJ. Protein modification by oxidants and the role of proteolytic enzymes. Biochem Soc Trans 1993;21:346-353.
46. Pacifici RE, Davies KJ. Protein, lipid and DNA repair systems in oxidative stress: The free-radical theory of aging revisited. Gerontology 1991;37:166-180.

47. Kritchevsky SB, Muldoon MF. Oxidative stress and aging: Still a hypothesis. J Am Geriatr Soc 1996;44:873-875.

48. Davies KJ. Oxidative stress: The paradox of aerobic life. Biochem Soc Symp 1995;61:1-31.

49. Pacifici RE, Davies KJ. Protein degradation as an index of oxidative stress. Methods Enzymol 1990;186:485-502.

50. Conconi M, Szweda L1, Levine RL et al. Age-related decline of rat liver multicatalytic proteinase activity and protection from oxidative inactivation by heat-shock protein 90. Arch Biochem Biophys 1996;331:232-240.

51. Tatsmo T, Stadtman FR, Floyd RA, Markesbery WR. Protein oxidation in aging brain. Ann NY Acad Sci 1992;663:110-119.

52. Stadtman ER. Metal-ion catalyzed oxidation of proteins: Biochemical mechanism and biological consequences. Free Radic Biol Med 1990;9:31532.5 .

53. Orr WC, Sohal RS. Relationship between antioxidants, prooxidants, and the aging process. Ann NY Acad Sci 1992;663:71-73.

54. Stadtman ER. Protein oxidation and aging. Science 1992:257:1220-1224.

55. Levine RL, Williams JA, Stadtman ER, Shacter E. Carbonyl assays for determination of oxidatively modified proteins. Methods Enzymol 1994;2.33:436-357.

56. Stadtman ER. The status of oxidatively modified proteins as a marker of aging. In: Esser K, Martin GM, eds. Molecular Aspects of Aging. New York: John Wiley \& Sons, 1995, pp 129-143.

57. Brownlee $\mathbf{M}$. Advanced protein glycosylation in diabetes and aging. Ann Rev Med 1995;46:223-234.

58. Monnier VM, Sell DR, Miyata S, Nagara RH. The Maillard reaction as a basis for a theory of aging. In: Finot PA, ed. Proceedings of the 4th International Symposium on the Maillard Reaction. Basel: Birkhausert-Verlag, 1993, pp 393-515.

59. Knecht KJ, Thorpe SR, Baynes JW. Role of oxygen in cross-linking and chemical modification of collagen by glucose. Diabetes 1992;41:42-48.

60. Wells-Knecht KJ, Blackledge JA, Lyons TJ et al. Glycation, glycoxidation, and cross-linking of collagen by glucose: Kinetics, mechanisms, and inhibition of late stages of the Maillard reaction. Diabetes 1994;43:676-683.

61. Wells-Knecht KJ, Zyzak DV, Litchfield JE et al. Mechanism of autoxidative glycosylation: Identification of glyoxaland arabinose as intermediates in the autoxidative modification of proteins by glucose. Biochemistry 1995;34:3702-3709.

62. Lee AT, Cerami A. Role of glycation in aging. Ann NY Acad Sci 1992;66.3:63-70.

63. Oimomi M, Kitamura Y, Nishimoto $S$ et al. Age-related acceleration of glycation of tissue proteins in rats. J Gerontol 1986;41:695-698.

64. Mikski I, Deyl Z. Change in the amount of epsilon-hexosyllysine UV absorbance and fluorescence of collagen with age in different animal species. J Gerontol 1991;46:B111-116.

65. Kohn RR, Cerami A, Monnier VM. Collagen aging in vitro by nonenzymatic glycosylation and browning. Diabetes 1984;33:57-59.

66. Garlick RL, Bunn HF, Spiro RG. Nonenzymatic glycation of basement membranes from human glomeruli and bovine sources: Effect of diabetes and aging. Diabetes 1988;37:1144-1155.

67. Tanaka S, Avigad G, Brodsky B, Eikenberry EF. Glycation induces expansion of the molecular packing of collagen. J Mol Biol 1988;203:495-505.

68. Brownlee $M$, Pongor $S$, Cerami A. Covalent attachment of soluble protein by nonenzymatically glycosylated collagen: Role in the in situ formation of immune complexes. J Exp Med 1983;158:1739-1744.

69. Sensi M, Tanzi B, Bruno RM, et al. Human glomerular basement membrane: Altered binding characteristics following in vitro non-enzymatic glycosylation. Ann N Y Acad Sci 1986;488:549-552.

70. Brownlee $M$, Vlassara $H$, Cerami A. Non-enzymatic glycosylation products of collagen covalently trap low-density lipoprotein. Diabetes 1985;34:938-941.

71. Tsilbary EC, Charonis AS, Reger LA et al. The effect of nonenzymatic glycosylation on the binding of the main noncollagenous NCI domain to type IV collagen. J Biol Chem 1988;26.3:4302-4308.

72. Charonis AS, Reger LA, Dege JE et al. Laminin alterations after in vitro nonenzymatic glycosylation. Diabetes 1990;39:807-814.

73. Hogan M, Cerami A, Bucala R. Advanced glycosylation end products block the antiproliferative effect of nitric oxide. J Clin Invest 1992;90:1110-1115.

74. Harding JJ, Beswick HT, Ajiboye R et al. Non-enzymic post-translation modifcation of proteins in aging: A review. Mech Ageing Dev 1989;50:7-16.

75. Patrick JS, Thorpe SR, Baynes JW. Nonenzymatic glycosylation of protein does not increase with age in human lenses. J Gerontol 1990;45:B18-23.

76. Perry RE, Swamy MS, Abraham EC. Progressive changes in lens crystallin glycation and high-molecular weight aggregate formation leading to a cataract 
development in streptozotocin-diabetes. Exp Eye Res 1987;44:269-282.

77. Makita $Z$, Vlassara H, Rayfield E et al. Hemoglobin-AGE: A circulating marker of advanced glycosylation. Science 1992;258:651-653.

78. Ganea E, Harding JJ. Inactivation of glucose-6-phosphate dehydrogenase by glycation. Biochem Soc Trans 1994;22:445S.

79. Ganea E, Harding JJ. Molecular chaperones protect against glycationinduced inactivation of glucose-6-phosphate dehydrogenase. Eur J Biochem 1995;231:181-185.

80. Heath MM, Rixon KC, Harding JJ. Glycation-induced inactivation of malate dehydrogenase protection by aspirin and a lens molecular chaperone, alpha-crystallin. Biochim Biophys Acta 1996;1315:176-184.

81. Shilton BH, Walton DJ. Sites of glycation of human and horse liver alcohol dehydrogenase in vivo. J Biol Chem 1991;266:5587-5592.

82. He RQ, Li YG, Wu XQ, Li L. Inactivation and conformational changes of the glycated and non-glycated D-glyceraldehyde-3-phosphate dehydrogenase during guanidine- $\mathrm{HCl}$ denaturation. Biochim Biophys Acta 995; 1253:47-56.

83. He RQ, Yang MD, Zheng $X$, Zhou JX. Isolation and some properties of glycated D-glyceraldehyde-3-phosphate dehydrogenase from rabbit muscle. Biochem J 1995;309:133-139.

84. Sun AQ, Yuksel KU, Rao GS, Gracy RW. Effects of active site modification and reversible dissociation on the secondary structure of triosephosphate isomerase. Arch Biochem Biophys 1992;295:421-428.

85. Sun AQ, Yuksel KU, Gracy RW. Relationship between the catalytic center and the primary degradation site of triosephosphate isomerase: Effects of active site modification and deamidation. Arch Biochem Biophys 1992;293:382-390.

86. Sun AQ, Yuksel KU, Gracy RW. Terminal marking of triosephosphate isomerase: Consequences of deamidation. Arch Biochem Bophys 1995;322:361-368.

87. Cini JK, Gracy RW. Molecular basis of the isozymes of bovine glucose-6phosphate isomerase. Arch Biochem Biophys 1986;249:500-505.

88. Geiger T, Clarke S. Deamidation, isomerization and racemization at asparaginyl and aspartyl residues in proteins. J Biol Chem 1987;262:785-794.

89. Clarke S. Propensity for spontaneous succinimide formation from aspartyl and asparaginyl residues in cellular proteins. Int J Pept Protein Res 1987;30:808-821.

90. Brunauer LS, Clarke S. Age-related accumulation of protein residues which can be hydrolyzed to D-aspartic acid in human erythrocytes. J Biol Chem 1986;261:12538-12543.

91. Ota IM, Ding L, Clarke S. Methylation at specific altered aspartyl and asparaginyl residues in glucagon by the erythrocyte protein carboxyl methyl transferase. J Biol Chem 1987;262:8522-8531.

92. Mcfadden PN, Clarke S. Protein carboxyl methyltransferase and methyl, acceptor proteins in aging and cataractous tissue of the human eye lens. Mech Ageing Dev 1986;34:91-105.

93. Rothstein $M$. The formation of altered enzymes in aging animals. Mech Ageing Dev 1979;9:197-202.

94. Rothstein M. Biochemical Approaches to Aging. New York: Academic Press, 1982, pp 213-25S.

95. Rothstein M. Age-related changes in enzyme levels and enzyme properties In: Rothstein M, ed. Review of Biological Research in Aging, Vol 2. New York: Alan R Liss, pp 421-433.

96. Sharma HK, Rothstein M. Age-related changes in the properties of enolase from Turbatrix aceti. Biochemistry 1978;17:2869-2876.

97. Sharma HK, Prasanna HR, Rothstein M. Altered phosphoglycerate kinase in aging rats. J Biol Chem 1980;255:5043-5050.

98. Sharma HK, Rothstein M. Altered brain phosphoglycerate kinase from aging rats. Mech Ageing Dev 1984;25:285-296.

99. Gafni A, Yuh KCM. Age-related molecular changes in skeletal muscle. In: Snyder DL, ed. Dietary Restriction and Aging. New York: Alan R. Liss, 1989, pp 277-282.

100. Hardt $H$, Rothstein $M$. Altered phosphoglycerate kinase from old rat muscle shows no change in primary structure. Biochim Biophys Acta 1985;831:13-21.

101. Yuh KCM, Gafni A. Reversal of age-related effects in rat muscle phosphoglycerate kinase. Proc Natl Acad Sci USA 1987;84:7458-7462.

102. Zuniga A, Gafni A. Age-related modifications in rat cardiac phosphoglycerate kinase: Rejuvenation of the old enzyme by unfolding-refolding. Biochim Biophys Acta 1988;955:50-57.

103. Cook LL, Gafni A. Protection of phosphoglycerate kinase against in vitro aging by selective cysteine methylation. J Biol Chem 1988;263:13991-13993.

104. Zhou JQ, White TP, Gafni A. Endurance-training induced changes in skeletal muscle phosphoglycerate kinase of old wistar rats. Mech Ageing Dev 1990:58:163-175
105. Kelly JW. Alternative conformations of amyloidogenic proteins govern their behavior. Curr Opin Struct Biol 1996;6:11-17.

106. Uemichi $T$, Liepnieks JJ, Benson MD. Hereditary renal amyloidosis with a novel variant fibrinogen. J Clin Invest 1994;93:731-736.

107. Cohen AS. Proteins of the systemic amyloidoses. Curr Opin Rheumatol 1994;6:55-67.

108. Kelly JW, Lansbury PT. A chemical approach to elucidate the mechanism of transthyretin and beta-protein amyloid fibril formation. Amyloid 1994;1:186-205.

109. O'Brien TD, Butler PC, Westermark P, Johnson KH. Islet amyloid polypeptide: A review of its biology and potential roles in the pathogenesis of diabetes mellitus. Vet Pathol 1993;30:317-332.

110. Edwards BJA, Morley JE. Amylin. Life Sci 1992;51:1899-1912.

111. Come JH, Lansbury Jr PT. Predisposition of prion protein homozygotes to Creutzfeldt-Jakob disease can be explained by a nucleation-dependent polymerization mechanism. J Am Chem Soc 1994;116:4109-4110.

112. Zhang $H$, Kaneko $K$, Nguyen JT et al. Conformational transitions in peptides containing two putative a-helices of the prion protein. J Mol Biol $1995 ; 250: 514-526$.

113. Nguyen J, Baldwin MA, Cohen FE, Prusiner SB. Prion protein peptides induce alpha-helice to beta sheet conformational transitions. Biochemistry 1995;34:4186-4192.

114. Lee JP, Stimson ER, Ghilardi JR et al. H-1 NMR of A-beta amyloid peptide congeners in water solution: Conformational changes correlate with plaque competence. Biochemistry 1995;34:5191-5200.

115. Simmons LK, May PC, Tomaaselli KJ et al. Secondary structure of amyloid $\beta$-peptide correlates with neurotoxic activity in vitro. Mol Pharmacol 1994:45:373-379.

116. Soto C, Frangione B. Two conformational states of the amyloid A-beta peptide: Implications for the pathogenesis of Alzheimer's disease. Neurosci Lett 199;186:115-118.

117. Howlett DR, Jennings KH, Lee DC et al. Aggregation state and neurotoxic properties of Alzheimer's beta-amyloid peptide. Neurodegen 1995;4:23-32.

118. Yanker BA, Dawes LR, Fisher $S$ et al. Neurotoxicity of a fragment of the amyloid precursor associated with Alzheimer's disease. Science $1989 ; 245: 417-420$.

119. Lorenzo A, Razzaboni B, Weir GC, Yankner BA. Pancreatic islet cell toxicity of amylin associated with type- 2 diabetes mellitus. Nature 1994;368:7\$6-760.

120. Charge SB, de-Koning EJ, Clark A. Effect of $\mathrm{pH}$ and insulin on fibrillogenesis of jslet amyloid polypeptide in vitro. Biochemistry 1995;34:14588-14593.

121. Cooper GJ, Day AJ, Willis AC et al. Amylin and the amylin gene: Structure, function and relationship to islet amyloid and to diabetes mellitus. Biochim Biophys Acta 1989;1014:247-258.

122. Blake CCF, Geisow MJ, Oatley S\}. Structure of prealbumin (transthyretin): Secondary, tertiary and quaternary interactions determined by Fourier refinement at 1.8 A. J Mol Biol 1978;121:339-356.

123. Colon W, Kelly JW. Partial denaturation of transthyretin is sufficient for amyloid fibril formation in vitro. Biochemistry 1992;31:8654-8660.

124. Lai Z, Colon W, Kelly JW. The acid-mediated denaturation of transthyretin proceeds through an intermediate that partitions into amyloid. Biochemistry 1996;35:6470-6482.

125. Hensley K, Butterfield DA, Matron M et al. A model for beta-amyloid aggregation and neurotoxicity based on the free radical generating capacity of the peptide: Implications of "molecular shrapnel" for Alzheimer's disease. Proc West Pharmacol Soc 1995;38:113-120.

126. Bhattacharya K, Glendening JM, Stopa $E$ et al. Advanced glycation end products contribute to amyloidosis in Alzheimer disease. Proc Natl Acad Sci USA 1994:91:4766-4770.

127. Horwitz J. Alpha crystallin can function as a molecular chaperone. Proc Natl Acad Sci USA 1992;89:10449-10453.

128. Hendrick JP, Hartl FU. Molecular chaperone functions of heat shock proteins. Annu Rev Biochem 1993;62:349-384.

129. Chrunyk BA, Evans J, Wetzel R. Probing the role of protein folding in inclusion body formation. In: Cleland JL, ed. Protein Folding in Vivo and in Vitro. Washington, DC: American Chemical Society, 1993, pp 46-58.

130. Heydari AR, Takahashi R, Gutsmann A et al. Hsp 70 and aging. Experientia 1994;50:1092-1098.

131. Pahlavani MA, Harris MD, Moore SA et al. The expression of heat shock protein 70 decreases with age in lymphocytes from rats and rhesus monkeys. Exp Cell Res 1995;218:310-318.

132. Kregel KC, Moseley PL, Skidmore R et al. Hsp 70 accumulation in tissues of heat-stressed rats is blunted with advancing age. J Appl Physiol 1995;79:1673-1678

133. Fargnoli J, Kunisada T, Fornace AJ et al. Decreased expression of heat shock protein $70 \mathrm{mRNA}$ and protein after heat treatment in cells of aged rats. Proc Natl Acad Sci USA 1990;87:846-850. 